\title{
Teste do Desempenho Escolar: evidências de validade do subteste de escrita
}

\author{
Claudia Hofheinz Giacomoni - Universidade Federal do Rio Grande do Sul. Porto Alegre, Brasil \\ Marcia de Lima Athayde - Universidade Federal do Rio Grande do Sul. Porto Alegre, Brasil \\ Cristian Zanon - Universidade São Francisco, Itatiba, Brasil \\ Lilian Milnitsky Stein - Universidade Federal do Rio Grande do Sul. Porto Alegre, Brasil
}

\begin{abstract}
Resumo
O Teste de desempenho escolar (TDE) (Stein, 1994) avalia, de maneira ampla, a aprendizagem por meio de três subtestes: leitura, escrita e aritmética. O objetivo deste estudo foi avaliar as propriedades psicométricas do subteste de escrita, a fim de avaliar sua estrutura interna, dificuldade e discriminação dos itens, utilizando métodos clássicos e modernos - Teoria de resposta ao item (TRI). A amostra foi composta de dados oriundos de pesquisas realizadas em quatro estados brasileiros ( $N=1831)$. Os resultados indicaram que o subteste de escrita apresenta: (a) evidências de unidimensionalidade, (b) está composto por itens de dificuldade média e (c) com alta capacidade de discriminação. Ademais, observou-se que o subteste discrimina satisfatoriamente estudantes entre primeira e terceira série e, menos adequadamente, estudantes de quarta, quinta e sexta série. Esse conjunto de resultados indica que o subteste apresenta evidências de validade, mas requer refinamento.

Palavras-chave: linguagem escrita; validade do teste; teoria de resposta ao item.
\end{abstract}

\section{School Achievement Test: evidence of validity of the writing subtest}

\begin{abstract}
The School achievement test (TDE) (Stein, 1994) assesses learning broadly through three subtests: reading, writing and arithmetic. The goal of this study was to evaluate the psychometric properties of the Writing Subtest, to evaluate the internal structure, items difficulty and discrimination, using Item response theory (IRT). The sample consisted of data from surveys conducted in four Brazilian states $(N=1831)$. Results indicated that the writing subtest presents: (a) evidence of unidimensionality, (b) is composed by items of average difficulty and (c) with high discrimination capability. Moreover, it was observed that the Subtests discriminate satisfactorily students between first and third grade and, less appropriately, students from fourth, fifth and sixth grade. This set of results indicates that the Subtest presents evidence of validity, but requires refinement. Keywords: written language; test validity; item response theory.
\end{abstract}

Test de Desempeño Escolar: evidencias de validez del sub-test de escritura

\begin{abstract}
Resumen
El Test de desempeño escolar (TDE) (Stein, 1994), evalúa de forma amplia el aprendizaje, por medio de tres Sub-test: Lectura, Escritura y Aritmética. El objetivo de este estudio fue evaluar las propiedades psicométricas del Sub-test de Escritura, para analizar su estructura interna, dificultad y discriminación de los ítems, utilizando métodos clásicos y modernos - Teoría de respuesta al ítem (TRI). La muestra consistió en datos originales de investigaciones realizadas en cuatro estados de Brasil (N=1831). Los resultados indicaron que el Sub-test de Escritura presenta: (a) evidencias de unidimensionalidad (b) se compone de ítems de dificultad media, y (c) alta capacidad de discriminación. Por otra parte, se observó que el sub-test discrimina de una manera satisfactoria estudiantes entre primer y tercer año, y de forma menos adecuada, estudiantes de cuarto, quinto y sexto año. Este conjunto de resultados indica que el Sub-test presenta evidencias de validez, pero requiere ser mejorado.

Palabras-clave: lenguaje escrito; validez del test; teoría de respuesta al ítem.
\end{abstract}

A aprendizagem da linguagem escrita é tema de interesse de diferentes profissionais, dentre eles fonoaudiólogos, pedagogos, psicopedagogos e psicólogos. Entender o processo envolvido no desenvolvimento dessa habilidade, bem como avaliar o domínio dos alunos nesse tópico, é fundamental para aprimorar os recursos de ensino, diagnóstico e tratamento.

A escrita pode ser considerada uma atividade mental complexa que, segundo o enfoque da Neuropsicologia e da Psicologia Cognitiva, envolve múltiplos processos interdependentes. A escrita envolveria dois processos fundamentais: a escrita de palavras e a de textos, sendo que a capacidade de escrever palavras não garante a competência narrativa e argumentativa do escritor (Salles, 2005).

Escrever não é apenas traçar letras que representam sons e palavras, mas sim compreender seus usos, funções, atribuir graus variados de significações (Zorzi, 2003). Aprender a língua escrita envolve diversos aspectos, dentre eles a ortografia, a relação entre letras e sons, e sua correspondência quantitativa, as variações entre o modo de pronunciar uma palavra e escrevê-la, a posição de cada letra no espaço gráfico, a direção da escrita, a linearidade e a segmentação (Zorzi, 1998). 
A linguagem escrita difere da linguagem oral em relação à sua aquisição. Enquanto que o desenvolvimento da linguagem oral é um processo natural para as pessoas que vivem em sociedade, a linguagem escrita requer uma aprendizagem formal, a qual, usualmente, ocorre no contexto escolar. Assim, a aprendizagem do código escrito é cultural, só se aprende pelo ensino (Zorzi, 2003).

A aprendizagem da linguagem escrita abrange os processos de alfabetização e letramento. $\mathrm{O}$ primeiro refere-se à capacidade de utilizar o sistema convencional de escrita, as habilidades de codificação e decodificação da língua escrita, identificando as relações fonema-grafema. Já o letramento abarca a imersão das crianças na cultura escrita, desenvolvendo habilidades de uso desse sistema, em atividades de leitura e escrita nas práticas sociais que envolvem a língua. A alfabetização e o letramento são processos interdependentes e indissociáveis (Soares, 2004).

A escrita alfabética representa a sequência fonológica das palavras, mas não o seu significado. É necessário que a criança compreenda o funcionamento desse código escrito, que se baseia na correspondência grafema-fonema, para alfabetizar-se (Nobile \& Barrera, 2009). O indivíduo necessita compreender uma série de propriedades do sistema alfabético, para poder vir a usar as letras com seus sons convencionais. Precisa entender que a escrita alfabética representa os segmentos orais das palavras, e não seus significados ou as características físicas dos objetos que elas nomeiam. Para registrar as palavras, escreve-se mais letras que as sílabas que pronunciamos. Há combinações ou sequências de letras permitidas e posições em que elas podem aparecer, além dos valores sonoros que podem assumir. Após todo esse aprendizado, os alunos tendem a apresentar muitas dúvidas sobre questões ortográficas envolvendo regras contextuais (exemplo: os empregos de "c" e "qu" em palavras como "quero" e "coisa") (Morais, 2007).

No processo de alfabetização, inicialmente, os aprendizes dominam as restrições ou propriedades do sistema de escrita alfabética e, só em seguida e aos poucos, vão internalizando a norma ortográfica (Morais, 2007). Todas as crianças apresentam "erros" nesse processo de apropriação da escrita, os quais vão diminuindo com o avanço da escolarização. Em geral, crianças seguem uma trajetória semelhante no desenvolvimento da ortografia, apresentando os mesmos tipos de erros (Zorzi, 1998). Desse modo, a apropriação do sistema ortográfico se dá de forma progressiva
(Nobile \& Barrera, 2009). As crianças, naturalmente, vão diminuindo os erros ortográficos, à medida que avançam nas séries escolares. Há erros que são esperados nas primeiras séries, mas não nas subsequentes (Moojen, 2009).

Além do papel da escolaridade e da idade no desenvolvimento linguístico, pode haver influência do sexo das crianças nesse desempenho na linguagem. Shaywitz et al. (1995) encontraram evidências de diferenças entre os sexos na organização funcional do cérebro para a linguagem, indicando que essas variações existem no nível do processamento fonológico. Harasty, Double, Halliday, Kril e McRitchie (1997) encontraram que mulheres apresentam as áreas de Wernicke e Broca, proporcionalmente, maiores que a dos homens, sendo que essa diferença anatômica poderia estar relacionada com as habilidades de linguagem superiores encontradas nas mulheres.

Parece existir uma lacuna de instrumentos cientificamente construídos para avaliação do desempenho escolar na realidade brasileira (Knijnik, Giacomoni, \& Stein, 2013). Nesse cenário, há um instrumento psicopedagógico que vem sendo largamente utilizado para esse propósito: o Teste de desempenho escolar (TDE) (Stein, 1994). Seu objetivo é avaliar crianças da $1^{a}$ a $6^{a}$ série do ensino fundamental, podendo ser utilizado com algumas reservas para $7^{\mathrm{a}}$ e $8^{\mathrm{a}}$ série. É comprovada sua larga utilização em estudos científicos, visto que vem sendo utilizado em pesquisas realizadas em todo o país e em diferentes áreas da ciência (Knijnik et al., 2013).

O TDE é composto por três subtestes que avaliam as capacidades básicas para o desempenho escolar: leitura, escrita e aritmética. A proposta desse instrumento é que os subtestes apresentem uma escala de itens em ordem crescente de dificuldade, os quais devem ser apresentados para as crianças independentemente de sua série. $\mathrm{O}$ teste pode ser interrompido, assim que os itens apresentados em determinado nível da escala forem impossíveis de serem resolvidos pelo examinando.

O subteste de Escrita foi concebido para investigar a capacidade básica envolvida na aprendizagem da habilidade em questão. Ele é composto por um ditado de 34 palavras contextualizadas e pela escrita do próprio nome (Stein, 1994) e avalia aspectos de escrita, como a conversão fonema-grafema e a ortografia. O instrumento não avalia os demais aspectos envolvidos na escrita.

Nesse contexto, o TDE permite a identificação de eventuais dificuldades na área da aprendizagem da 
linguagem escrita. O diagnóstico do nível do desempenho em escrita das crianças é importante, para verificar se ele encontra-se adequado para a idade e série escolar do aprendiz. Além disso, é necessário verificar se o aprendiz apresenta dificuldades na aprendizagem da escrita, pois isso viabilizaria a criação de métodos de ensino que favoreçam o desenvolvimento dessa competência.

A aplicação do TDE permite averiguar as áreas nas quais a criança possa estar apresentando dificuldades, distinguindo a escrita da leitura e da aritmética. Segundo a autora do teste (Stein, 1994), a partir dessa constatação, o examinador poderá aplicar uma avaliação mais específica na área defasada, buscando elucidar em que aspectos residem as dificuldades da criança.

Foram encontrados três estudos que verificaram as características psicométricas do TDE (Lúcio \& Pinheiro, 2012; Lúcio, Pinheiro, \& Nascimento, 2009; Ferreira et al., 2012). Essas pesquisas apontaram a necessidade de revisar o instrumento em questão. Esses estudos encontraram lacunas no TDE, como a distribuição desproporcional no nível de dificuldade de cada item, a pouca distinção entre alunos com alto e baixo desempenho escolar, além da desatualização das normas (Knijnik et al., 2013).

Ainda, a mudança no cenário educacional brasileiro, aumentando o período do ensino fundamental para nove anos, é outro fator determinante para a necessidade de atualização do TDE. Essas modificações reformulam e redistribuem os conteúdos do ensino fundamental, fazendo com que o TDE esteja desatualizado no que se refere aos dados normativos para cada série (Knijnik et al., 2013).

Portanto, o objetivo do presente estudo foi o de verificar evidências de validade do subteste de Escrita. Mais especificamente, pretendeu-se investigar: a) estrutura interna do subteste; b) avaliar o grau de dificuldade e discriminação dos itens através de Teoria de resposta ao item (TRI); e c) avaliar as diferenças de habilidade de escrita nas diferentes séries e entre os sexos. Uma vez que o subteste de Escrita foi criado para avaliar somente as habilidades de conversão fonema-grafema e ortografia, evidências de unidimensionalidade dos escores corroborariam essa suposição. Considerando que os itens do subteste deveriam ser apresentados em uma ordem crescente de dificuldade, espera-se que os índices de dificuldade estimados pela TRI indiquem que a ordem apresentada seja adequada. Por fim, espera-se que existam diferenças de habilidade de escrita entre todas as séries estudadas.

\section{Método}

\section{Participantes}

A amostra estudada foi composta por 1831 estudantes (45,6\% meninas) provindas de escolas municipais $(66,8 \%)$, estaduais $(17,7 \%)$, escola pública não especificada $(9,3 \%)$ e escola particular $(6,2 \%)$. Os participantes pertenciam aos estados de Santa Catarina (42,1\%), São Paulo (28,1\%), Rio Grande do Sul (17,4\%) e Paraíba (12,4\%). Em relação às séries, aproximadamente, 9,7\% dos estudantes frequentavam a primeira; $36,3 \%$ a segunda; $22 \%$ a terceira; $19,5 \%$ a quarta; $8,5 \%$ a quinta e $4 \%$ a sexta série.

\section{Instrumento}

Foram utilizados os dados do TDE, oriundos de pesquisas realizadas nos últimos dez anos com esse instrumento.

\section{Procedimento de coleta de dados}

A partir do levantamento acerca das publicações utilizando o TDE desde seu lançamento, (Knijnik et al., 2013) foi feito contato com alguns dos pesquisadores responsáveis pelas pesquisas encontradas, a fim de solicitar autorização para utilização dos seus dados do TDE. Contataram-se pesquisadores cujas coletas foram realizadas nos últimos dez anos. Seguindo a orientação do Comitê de Ética para procedimentos no compartilhamento de dados, aqueles pesquisadores que concordaram em ceder seus dados assinaram um Termo de Compromisso de Dados, também assinado pelas pesquisadoras responsáveis pelo presente estudo.

\section{Procedimento de análise dos dados}

Para investigar a estrutura interna do subteste Escrita, realizou-se uma análise de eixos principais. Encontradas evidências de unidimensionalidade para o subteste, requisito para análises de TRI, procedeu-se às estimações dos parâmetros dos itens e do nível de habilidade dos participantes usando TRI. Por fim, realizou-se análises de variância para avaliar diferenças nos escores de escrita da primeira a sexta série e teste T para investigar possíveis diferenças de sexo. Foram utilizados os escores estimados pela TRI como variáveis dependentes na ANOVA e teste $\mathrm{T}$.

As análises de TRI foram conduzidas no programa BILOG-MG (Zimowski, Muraki, Mislevy, \& Bock, 1996) e o modelo escolhido foi o de dois parâmetros. Esse modelo foi selecionado por apresentar melhor ajuste que $\mathrm{o}$ modelo de um parâmetro. $\mathrm{O}$ ajuste dos 
modelos foi avaliado pelos resíduos padronizados. O de três parâmetros não foi considerado por ser improvável que os testandos acertem itens por acaso, uma vez que o formato do subteste não fornece alternativas. O método de estimação usado foi o maximum likelihood.

\section{Resultados}

Análise da estrutura interna

Para avaliar a dimensionalidade do subteste de Escrita, submeteu-se o conjunto de 34 itens a uma análise de eixos principais. Verificou-se KMO de 0,98 e teste de esfericidade de Bartlett significativo, o que indica adequação dos dados para a realização da análise. Observou-se um fator predominante, mais de quatro vezes maior que o segundo, o que indica evidências de unidimensionalidade. O primeiro eigenvalue foi de 12,58 e explicou $37 \%$ da variância, enquanto o segundo fator explicou $6 \%$ da variância e apresentou um eigenvalue de 2,17. A solução de um fator apresentou cargas fatoriais que variaram de 0,33 a 0,74 .

\section{Dificuldade e discriminação dos itens}

Para estimação da dificuldade e discriminação dos itens, procedeu-se à análise de TRI. Como observado na Tabela 1, a maioria dos itens apresenta dificuldade média, sendo que a média geral de dificuldade dos itens foi $0,23(D P=0,74)$. Ainda, os resultados evidenciaram que os índices de discriminação dos itens são elevados $(M=1,4 ; D P=0,3)$. Em outras palavras, pode ser observado que a grande maioria dos itens é de dificuldade média (entre -1 e 1) e avaliam, adequadamente, sujeitos com níveis médios de habilidade, mas não avaliam, de forma adequada, sujeitos com baixa ou alta habilidade.

Outro ponto que merece nota é que a ordem de dificuldade apresentada pelo processo de estimação baseado na TRI difere da sequência sugerida por experts, quando se constituiu o teste. Por exemplo: a) os itens 3 e 13 apresentaram níveis de dificuldade inferior ao item 1 que deveria ser o mais fácil; b) os itens 27 e 33 apresentaram a mesma dificuldade; c) o item 6 deveria estar entre os mais fáceis, no entanto apresentou-se entre os de maior dificuldade (nível médio).

Comparacõos de séries e sexo

Diferenças entre as séries escolares também foram verificadas $F(5,1844)=173,95 ; p<0,001$ e analisadas com testes post hoc - Scheffe - utilizando-se como critério de significância estatística $(\phi<0,05)$. Mais especificamente, observou-se que os participantes da
Tabela 1. Dificuldade e Discriminação dos Itens do Subteste Escrita Estimados pelo Modelo de Dois Parâmetros da TRI

\begin{tabular}{|c|c|c|}
\hline Item & Discriminação & Dificuldade \\
\hline $\mathrm{D} 03$ & 0.717 & -1.440 \\
\hline D13 & 1.019 & -1.326 \\
\hline D04 & 1.520 & $-0.848^{a}$ \\
\hline D01 & 1.520 & $-0.706^{a}$ \\
\hline D02 & 1.641 & $-0.691^{\mathrm{a}}$ \\
\hline D10 & 1.230 & $-0.632^{a}$ \\
\hline D11 & 1.499 & $-0.532^{\mathrm{a}}$ \\
\hline D07 & 1.725 & $-0.495^{a}$ \\
\hline D05 & 1.814 & $-0.342^{\mathrm{a}}$ \\
\hline D12 & 1.166 & $-0.324^{a}$ \\
\hline D14 & 1.632 & $-0.008^{a}$ \\
\hline D08 & 2.061 & $0.046^{a}$ \\
\hline D09 & 2.062 & $0.227^{\mathrm{a}}$ \\
\hline D17 & 1.884 & $0.258^{\mathrm{a}}$ \\
\hline D20 & 1.109 & $0.269^{\mathrm{a}}$ \\
\hline D18 & 1.270 & $0.287^{a}$ \\
\hline D21 & 1.552 & $0.343^{a}$ \\
\hline D22 & 1.138 & $0.361^{\mathrm{a}}$ \\
\hline D15 & 1.094 & $0.431^{\mathrm{a}}$ \\
\hline D16 & 1.677 & $0.463^{a}$ \\
\hline D31 & 1.788 & $0.486^{a}$ \\
\hline D24 & 1.097 & $0.562^{\mathrm{a}}$ \\
\hline D23 & 1.732 & $0.564^{a}$ \\
\hline D28 & 1.521 & $0.599^{\mathrm{a}}$ \\
\hline D30 & 1.337 & $0.735^{\mathrm{a}}$ \\
\hline D19 & 1.358 & $0.818^{\mathrm{a}}$ \\
\hline D25 & 1.305 & $0.834^{a}$ \\
\hline D27 & 1.091 & $0.842^{\mathrm{a}}$ \\
\hline D33 & 0.964 & $0.843^{\mathrm{a}}$ \\
\hline D34 & 1.675 & $0.997^{\mathrm{a}}$ \\
\hline D29 & 1.898 & 1.004 \\
\hline D26 & 1.719 & 1.066 \\
\hline D06 & 1.013 & 1.383 \\
\hline D32 & 1.309 & 1.609 \\
\hline $\mathrm{M}(\mathrm{dp})$ & $1.4(0.3)$ & $0.23(0.74)$ \\
\hline
\end{tabular}


primeira, segunda e terceira série diferem, significativamente, entre si e, também, dos participantes da quarta, quinta e sexta série. Os estudantes da quarta série não diferem, significativamente, dos da quinta, mas diferem dos da sexta. Por fim, os estudantes da quinta série não diferem dos estudantes da sexta. Apesar de algumas séries não apresentarem diferenças estatisticamente significativas, a análise dos tamanhos de efeitos revelam que há pequenas diferenças de efeito entre essas séries. A Tabela 2 apresenta as médias, desvios-padrão e tamanhos de efeito (d de Cohen) das diferenças de habilidades de escrita de acordo com as séries dos participantes. O d de Cohen é uma estimativa em termos de desvio-padrão, ou seja, os pequenos efeitos verificados entre as séries que não apresentaram diferença significativa indicaram, pelo menos, 0,3 desvio-padrão.

Em relação às diferenças da primeira série com as demais, observam-se grandes disparidades em termos de tamanho de efeito. Por exemplo: a diferença entre a primeira e segunda série é de, aproximadamente 1, desvio-padrão (grande diferença), sendo que ela aumenta gradualmente de acordo com a série. Esse aumento é esperado, já que estudantes de séries mais avançadas deviam apresentar maiores habilidades na escrita. A diferença entre a primeira e sexta série é de 3,6 desvios-padrões, ou seja, é uma diferença muito grande de habilidade de escrita. Pode-se, ainda, verificar efeitos moderados entre terceira e quarta série $(d=0,58)$, e entre a quinta e a sexta série $(d=0,55)$, o que indica diferenças de mais de 0,5 desvio-padrão entre esses grupos. Conjuntamente, esses dados sugerem que o subteste de Escrita consegue discriminar participantes de diversas séries e indica que, de forma geral, o tamanho das diferenças é elevado com o aumento da distância entre as séries comparadas. Quanto à variável sexo, foi verificada uma diferença significativa entre meninos $(M=-0,15$, $D P=1,15)$ e meninas $(M=0,03, D P=1,15) ; t(1846)=3,5$; $p<0,001$. A diferença dos grupos apresentou um efeito pequeno $(d=0,16)$, mas indica que, de modo geral, as meninas apresentam habilidades de escrita superiores às dos meninos.

\section{Discussão}

O objetivo deste estudo foi investigar evidências de validade no subteste de Escrita do TDE. Nesse sentido, verificou-se que a estrutura interna do subteste apresenta um fator predominante que indica a unidimensionalidade do construto. Isso sugere que a variável latente Habilidade de escrita é a responsável pela variância dos escores dos participantes.

Diferentemente do esperado, a estimação da dificuldade dos itens pela TRI revelou que a ordem de dificuldade adotada pelo manual do subteste não é a mais apropriada. Na Tabela 1, os itens estão dispostos em ordem de dificuldade estimada pela TRI. Como se pode ver, vários são os itens que estão "fora de lugar", o que indica a necessidade de revisão da ordem de apresentação dos itens no caderno do teste. Esse achado sugere que a instrução dada ao participante para "interromper o ditado após dez erros consecutivos, passando, então, para o subteste seguinte" deve ser flexibilizada, sob pena de comprometer a aplicação e interpretação dos resultados.

Tabela 2. Estatísticas Descritivas e Tamanhos de Efeito das Diferenças de Habilidade de Escrita entre as Séries

\begin{tabular}{|c|c|c|c|c|c|c|c|c|}
\hline \multirow{2}{*}{ séries } & \multicolumn{3}{|c|}{ Descritivas } & \multicolumn{5}{|c|}{ Efeito ( $d$ de cohen) entre as séries } \\
\hline & $\mathrm{N}$ & média & $\mathrm{Dp}$ & $1^{a}$ série & $2^{a}$ série & $3^{a}$ série & $4^{a}$ série & $5^{a}$ série \\
\hline $1^{\mathrm{a}}$ & 180 & $-1,54$ & 1,21 & - & & & & \\
\hline $2^{\mathrm{a}}$ & 672 & $-0,38$ & 1,10 & 1,01 & - & & & \\
\hline $3^{\mathrm{a}}$ & 407 & 0,06 & 0,95 & 1,48 & 0,35 & - & & \\
\hline $4^{a}$ & 360 & 0,53 & 0,65 & 2,22 & 0,56 & 0,58 & - & \\
\hline $5^{a}$ & 157 & 0,74 & 0,64 & 2,46 & 1,29 & 0,78 & 0,34 & - \\
\hline $6^{a}$ & 74 & 1,06 & 0,50 & 3,60 & 1,80 & 1,26 & 0,93 & 0,55 \\
\hline total & 1850 & $-0,07$ & 1,15 & & & & & \\
\hline
\end{tabular}


Observou-se, também, um predomínio de itens de dificuldade média, o que permite a avaliação adequada apenas de sujeitos com níveis médios de habilidade. Esse achado vai de encontro com o que sugere Pasquali (2010), visto que o desejável seria que a distribuição dos itens, em termos de níveis de dificuldade, se aproximasse da distribuição normal, com uma menor proporção de itens fáceis e difíceis. Contudo, vale ressaltar que, nesse caso, faltam itens fáceis e difíceis no teste.

De acordo com as expectativas apresentadas, verificou-se que o desempenho dos participantes aumenta de acordo com a escolaridade. Também, observou-se que o subteste de Escrita consegue discriminar participantes de diferentes séries, sendo que essa diferença é, predominantemente, mais acentuada entre grupos de séries mais distantes, por exemplo: primeira e sexta, segunda e sexta, terceira e quinta série. As diferenças entre segunda e terceira, e entre quinta e sexta série, são pequenas, o que indica que esse instrumento tem menor capacidade de diferenciar esses grupos. As diferenças observadas para estudantes de quarta, quinta e sexta série não são estatisticamente significativas, mas indicam que o teste tem capacidade de discriminação entre as séries (ver tamanhos de efeito, Tabela 2), já que há diferença pequena (entre quarta e quinta), moderada (entre quinta e sexta) e grande (entre quarta e sexta). O fato das diferenças entre esses grupos não terem sido estatisticamente significativas, possivelmente, deve-se ao número reduzido de estudantes nesses grupos, em relação aos demais. Mesmo assim, esses resultados indicam que o subteste precisa de mais itens com dificuldades variadas e com conteúdos específicos às séries estudadas. Essa revisão é essencial, já que houve mudanças curriculares desde a construção do TDE, e possibilitarão maior discriminação, especialmente, entre os grupos que apresentaram pequenas diferenças.

De forma geral, esses resultados vão ao encontro do que era esperado, visto que as crianças apresentam melhor desempenho na ortografia, na medida em que progridem no nível de escolaridade. Quanto menor a série, maior é a quantidade de erros (Moojen, 2009).

Esse achado, entretanto, coincide com o que é comumente encontrado na literatura. Ainda, para Pedroso e Rotta (2006), os transtornos da linguagem incidem em dois a quatro meninos para cada menina, sendo que as meninas, em geral, adquirem a linguagem antes que os meninos. Wertzner e Oliveira (2002), também, observaram resultado semelhante, visto que, em seus achados, o distúrbio fonológico ocorreu em maior proporção no sexo masculino.
$\mathrm{Na}$ pesquisa de Flores-Mendoza, Mansur-Alves, Lelé e Bandeira (2007), as autoras verificaram que as meninas apresentaram melhores resultados do que os meninos no escore total do TDE e nos subteste de Escrita e Leitura. Os meninos saíram-se melhor apenas no subteste de Aritmética.

Entretanto, não há consenso na literatura de que as meninas apresentam melhor desempenho em tarefas de linguagem. Na pesquisa de Athayde, Baesso, Dias, Giacchini e Mezzomo (2009), as autoras encontraram maior precisão fonológica por parte dos meninos. Igualmente, Mota, Athayde e Mezzomo (2008) observaram que as crianças do sexo feminino apresentaram, em média, mais erros e demandaram mais tempo para a realização de prova de nomeação rápida. Por outro lado, Befi-Lopes, Caceres e Araújo (2007) não encontraram diferenças significativas entre os sexos quanto ao uso de substantivos e verbos. Há ainda a pesquisa de Marturano, Toller e Elias (2005), que aplicou o TDE em crianças com queixa de desempenho escolar pobre. As autoras também não encontraram diferenças de sexo nos resultados do TDE. Portanto, Athayde et al. (2009) referem não haver resultados conclusivos a respeito da variável sexo, visto que a natureza das diferenças das habilidades entre homens e mulheres ainda não está clara.

\section{Referências}

Athayde, M. L., Baesso, J. S., Dias, R. F., Giacchini, V., \& Mezzomo, C. L. (2009). O papel das variáveis extralinguísticas idade e sexo no desenvolvimento da coda silábica. Revista da Sociedade Brasileira de Fonoaudiologia, 14(3), 293-9.

Befi-Lopes, D. M., Caceres, A. M., \& Araujo, K. (2007). Aquisição de verbos em pré-escolares falantes do português brasileiro. Revista CEFAC, 9(4), 444-452.

Ferreira, F. L., Costa, D. S., Micheli, L. R.,Oliveira, L. F., Pinheiro-Chagas, P., \& Haase, V. G. (2012). School achievement test: Normative data for a representative sample of elementary school children. Psychology \& Neuroscience, 5(2), 157-164.

Flores-Mendoza, C. E., Mansur-Alves, M., Lelé, A. J., \& Bandeira, D. R. (2007). Inexistência de diferenças de sexo no fator $g$ (inteligência geral) e nas habilidades específicas em crianças de duas capitais brasileiras. Psicologia: Reflexão e Crítica, 20(3), 499-506.

Psico-USF, Bragança Paulista, v. 20, n. 1, p. 133-140, jan./abr. 2015 
Harasty J., Double K. L., Halliday G.M., Kril J. J., \& McRitchie D. A. (1997). Language-associated cortical regions are proportionally larger in the female brain. Archives of Neurology, 54(2), 171-6.

Knijnik, L. F., Giacomoni, C., \& Stein, L. M. (2013). Teste de desempenho escolar: Um estudo de levantamento (Manuscrito submetido para publicação).

Lúcio, P. S., \& Pinheiro, A. M. V. (2012). Novos estudos psicométricos para o Ssubteste de leitura do teste de desempenho escolar (Manuscrito submetido para publicação).

Lúcio, P. S., Pinheiro, A. M. V., \& Nascimento, E. (2009). O impacto da mudança no critério de acerto na distribuição dos escores do subteste de leitura do teste de desempenho escolar. Psicologia em Estudo, 14(3), 593-601.

Marturano, E. M., Toller, G. P., \& Elias, L. C. S. (2005). Gênero, adversidade e problemas socioemocionais associados à queixa escolar. Estudos de Psicologia 22(4), 371-380.

Moojen, S. M. P. (2009). Linguagem escrita. Em S. M. P. Moojen (Ed.), A escrita ortográfica na escola e na clínica. Teoria, avaliação e tratamento (pp. 19- 40). São Paulo: Casa do Psicólogo.

Morais, A. G. (2007). A norma ortográfica do português: o que é? Para que serve? Como está organizada? Em Silva, A., Morais, A. G., \& Melo, K. L. R. (Orgs.), Ortografia na sala de aula. (11-28). Belo Horizonte: Autêntica.

Mota, H. B., Athayde, M. L., \& Mezzomo, C. L. (2008). $\mathrm{O}$ acesso ao léxico em crianças com desenvolvimento fonológico normal e desviante. Letras de Hoje, 43(3), 54-60.

Nobile, G. G., \& Barrera, S. D. (2009). Análise de erros ortográficos em alunos do ensino público fundamental que apresentam dificuldades na escrita. Psicologia em Revista, 15(2), 36-55.
Pasquali, L. (2010). Instrumentação psicológica: Fundamentos epráticas. Porto Alegre: Artmed.

Pedroso, F.S.; Rotta, N.T. (2006) Transtornos da linguagem. Em N. T. Rotta, \& R. S. Riesgo. (Org.) Transtornos da aprendizagem-Abordagem neurobiologica e multidisciplinar. (pp. 131-148). Porto Alegre: Artmed.

Salles, J. F. (2005). Habilidades e dificuldades de leitura e escrita em crianças de $2^{a}$ série: Abordagem neuropsicológica cognitiva (Tese não publicada). Universidade Federal do Rio Grande do Sul, Rio Grande do Sul.

Shaywitz, B. A., Shaywitz, S. E., Pugh, K. R., Constable, R. T., Skudlarski, P., \& Fulbright, R. K. (1995). Sex differences in the functional organization of the brain for language. Nature, 373(6515), 607-609.

Soares, M. (2004). Letramento e alfabetização: As muitas facetas. Revista Brasileira de Educação, (25), 5-17.

Stein, L. M. (1994). TDE - Teste de desempenho escolar: Manual para aplicação e interpretação. São Paulo: Casa do Psicólogo.

Wertzner, H. F., \& Oliveira, M. M. F. (2002) Semelhanças entre os sujeitos com distúrbio fonológico. Revista Pró-Fono, 14(2) 143-52.

Zimowski, M. F., Muraki, E., Mislevy, R. J. \& Bock, R. D. (1996). BILOG-MG: Multiple-group IRT analysis and test maintenance for binary items. Chicago: Scientific Software, Inc.

Zorzi, J. L. (1998). Aprender a escrever: A apropriação do sistema ortográfico. Porto Alegre.

Zorzi, J. L. (2003) Aprendiragem e distúrbios da linguagem escrita - Questões clínicas e educacionais. Porto Alegre.

Recebido em: 13/12/2013

Reformulação em: 13/04/2014

Aprovado em: 23/06/2014 
Nota dos autores:

Esta pesquisa foi financiada pela CAPES.

Sobre os autores:

Claudia Hofheinz Giacomoni é graduada em Psicologia pela PUCRS (1995), mestre em Psicologia do Desenvolvimento pela UFRGS (1997), doutora em Psicologia do Desenvolvimento pela UFRGS (2002) e professora adjunta do departamento de Psicologia do Desenvolvimento e da Personalidade da UFRGS.

Marcia de Lima Athayde é fonoaudióloga da UFRGS, graduada em Fonoaudiologia pela UFSM (2006), mestre em Distúrbios da Comunicação Humana pela UFSM (2009) e doutoranda em Psicologia - Processos Cognitivos, pela PUCRS. E-mail: fga.marcia@yahoo.com.br

Cristian Zanon é graduado em Psicologia pela UFSM (2006), mestre (2009) e doutor (2011) em Psicologia pela UFRGS, e professor no programa de pós-graduação em Psicologia da Universidade São Francisco, Itatiba. E-mail: cristianzanon@yahoo.com.br

Lilian Milnitsky Stein é graduada em Psicologia pela UFRGS (1983), especialista em Psicologia Escolar pela PUCRS (1985), mestre em Applied Cognitive Science pelo Ontario Institute For Studies In Education (1989), doutora em Cognitive Psychology pela University of Arizona (1998) e professora titular do programa de pós-graduação em Psicologia da PUCRS. E-mail: lilian@pucrs.br

Contato com os autores:

Claudia Hofheinz Giacomoni

Rua Ramiro Barcelos, 2600, sala 124. Santa Cecília. Porto Alegre/RS

CEP: 90035-003

E-mail: giacomon@uol.com.br

Telefone: (051) 9919-1809 